\title{
Cambios y malnutrición: problemas alimentarios en San Andrés de Tupicocha
}

\author{
Malnutrition and changes: nutritional problems in San Andrés de Tupicocha
}

\author{
Juan Andrés Gómez de la Torre Barúa ${ }^{1 *}$; María Nilda Varas Castrillo²
}

* Autor de correspondencia

\begin{abstract}
Resumen
El objetivo del presente estudio es ampliar el entendimiento sobre el concepto de malnutrición, a partir de información proveniente de un trabajo de campo etnográfico que se realiza en San Andrés de Tupicocha, ubicado en la provincia de Huarochirí, región Lima, entre los meses de enero y septiembre del 2015. El estudio otorga varios testimonios donde los entrevistados perciben efectos en la nutrición familiar, a partir de dos cambios: 1) un cambio en el aumento del acceso al agua para riego; y 2) otro cambio en los productos agrícolas, que antes cultivaban para el consumo familiar, ahora se cultivan para la comercialización. Así, el estudio entrega como resultado una relación entre la construcción de un nuevo sistema de riego - localmente llamadas represas comunales -, la producción agrícola de las familias y su alimentación. Con este análisis surge la necesidad de incluir nuevos factores para entender la situación de la malnutrición en Tupicocha, concluyendo que se percibe a estas represas como fuentes de cambio, alterando prácticas productivas con agricultores cultivando productos con mayor valor comercial, dejando de cultivar productos con mayor valor nutricional.
\end{abstract}

Palabras clave: Malnutrición; Alimentación; Mitos sobre alimentación; Sistema de riego; Represas comunales; Innovaciones; Cambios; Efectos.

\begin{abstract}
The objective of this stady is to broaden the understanding of the concept of malnutrition, based on information from an ethnographic fieldwork carried out in San Andrés de Tupicocha, located in the province of Huarochirí, Lima region, between the months of January and September 2015. The study provides several testimonies where the interviewees perceive effects on family nutrition, based on two changes: 1) a change in the increase in access to water for irrigation; and 2) another change in agricultural products which were previously cultivated for family consumption, and are now cultivated for commercialization. Thus, the study gives as one of its results that there is a relationship between the construction of a new irrigation system - locally called communal dams -, the agricultural production of families and their food. With this analysis arises the need to include new factors to understand the situation of malnutrition in Tupicocha, concluding that these dams are perceived as sources of change, altering productive practices with farmers growing products with greater commercial value, no growing, anymore, products with greater nutritional value.
\end{abstract}

Keywords: Malnutrition; Food; Myths about food; Irrigation system; Communal dams; Innovations; Changes; Effects.

\section{Introducción}

Sobre la base del estudio realizado en la tesis titulada "Innovación, agua y medios de vida: discursos de cambio en pobladores de San Andrés de Tupicocha", elaborada por Gómez de la Torre (2017), el texto aquí sostienen tres premisas. La primera manifiesta que la llegada de un nuevo sistema de riego - llamada de represas comunales - influyó en el cambio de la producción agrícola local, entendiendo esta como segunda premisa. La última premisa advierte, entonces, que estos cambios han alterado la alimentación familiar, en tanto que los productores ya no quieren cultivar productos para el autoconsumo, a la vez cambiando su dieta, mientras la producción agraria ahora se comercializa en los mercados de la capital, Lima. Se llevó a cabo una evaluación en el 2013, financiado por el BID y su Fondo Multilateral de Inversiones (FOMIN) en donde ninguno de los resultados (éxitos o dificultades), ni los aprendizajes, tenían en cuenta la situación nutricional de la cuenca baja, media y alta de Lurín (Jurado, 2013). Estos factores influenciarían el estado nutricional de las niñas y niños de Tupicocha.

Según información provista por el MIDIS (2012), Tupicocha tiene un $75 \%$ de niñas y niños con Desnutrición Crónica Infantil (DCI), una de las formas de malnutrición. El Perú sigue siendo un país que se enfrenta a la "malnutrición". La (OMS, 2018) ha definido este concepto

\footnotetext{
${ }^{1}$ Licenciado en Antropología (PUCP), Mágister en Innovación Agraria para el Desarrollo Rural (UNALM). Email: jgomezdelatorrebarua@yahoo. com

${ }^{2}$ Doctora en Ciencias Ambientales, docente Principal, Universidad Nacional Agraria La Molina. Email: nvaras@lamolina.edu.pe
} 
en base a tres dimensiones:

- "La desnutrición, que incluye la emaciación (un peso insuficiente respecto de la talla), el retraso del crecimiento (una talla insuficiente para la edad) y la insuficiencia ponderal (un peso insuficiente para la edad).

- Las carencias de micronutrientes (la falta de vitaminas o minerales importantes) o el exceso de micronutrientes.

- El sobrepeso, la obesidad y las enfermedades no transmisibles relacionadas con la alimentación como las cardiopatías, la diabetes y algunos cánceres (OMS, 2018)."

La Desnutrición Crónica Infantil (DCI) ha sido uno de los problemas más serios a los que el país se ha venido enfrentando. Este mal ha sido considerado por múltiples instituciones como uno de los principales causantes que impide romper con el ciclo de la pobreza: limita el crecimiento y desarrollo corporal, cognitivo, emocional y social de las niñas y niños que sufren con este mal (Del Pino et al., 2012).

En un texto elaborado por el Banco Internacional de Reconstrucción y Fomento (IBRD por siglas en inglés [parte del Grupo Banco Mundial]), dan cuenta del avance logrado por el Perú en la lucha contra la malnutrición en los últimos diez años (2006-2016). Según los autores del texto, este avance se ha dado en varios frentes: 1) con los padres de familia que tenían niñas y niños menores de 5 años; 2) con el personal de salud que atendía a estos padres de familia en sus localidades - especialmente aquellos situados en zonas rurales -; y 3) con actores políticos a nivel nacional, regional, provincial y distrital.

Particularmente con los padres de familia, los autores plantean que este grupo pensaba que sus hijos crecían bien, cuando no lo estaban haciendo. A su vez, el texto sostiene que el personal de salud no venía cumpliendo cabalmente sus tareas para detener o erradicar la Desnutrición Crónica Infantil (INEI, 2009). Finalmente, no había compromiso político para luchar contra la DCI, evidenciando la falta de un enfoque político sobre este mal. Manifestaron que para comenzar a luchar contra la malnutrición, estos aspectos tenían que cambiar. Hoy en día, logrados estos cambios, los avances demuestran diferencias notorias en los indicadores (Marini y Rokx, 2017).

El Ministerio de Salud (MINSA), por su parte, recopiló sobre malnutrición (DCI, sobrepeso, anemia) información entre el 2010 y 2016. Se construyó una secuencia histórica de datos en donde el $23,2 \%$ de todos las niñas y niños (casi uno de cada cuatro niñas y niños) del país sufrían de algún tipo de desorden alimenticio para el 2010; para el 2014, se había reducido a $14.6 \%$ del total. Esta información sostiene lo propuesto por Marini y Rokx (2017).

No hay duda que, hasta ahora, muchas niñas y niños en todo el territorio nacional siguen sufriendo de alguna forma de malnutrición, en especial aquello relacionado a la DCI. Si se añade, además, la situación sobre obesidad y anemia, en niños y niñas menores de 5 años, hay que reconocer la complejidad inherente al tema. Esta misma complejidad justifica la necesidad de continuar las indagaciones sobre otros factores que inciden en sostener y propagar la malnutrición.

El texto aquí elaborado plantea como objetivo ahondar en la relación compleja y multidimensional que Del Pino et al. (2012) mencionan en su libro sobre la malnutrición: hay que entender estos problemas más allá de las meras prácticas nutricionales que se desarrollan entre madre e hijos (como sí lo establecen Marini y Rokx, 2017). Se parte de la información recopilada en el trabajo de Gómez de la Torre (2017) para profundizar esta relación, tomando en consideración las tres premisas encontradas por el autor de la tesis durante su estancia en la zona. A la par se manifiesta un cambio importante por parte de la población local: su dieta ha sostenido un cambio, en tanto que los productos que anteriormente usaban para alimentarse, hoy en día ya no se cosechan.

\section{Materiales y métodos}

Durante los meses de febrero a agosto del 2015, se desarrolló una serie de visitas, junto con una estancia prolongada (de un mes), en la capital del distrito de San Andrés de Tupicocha. Durante esta estancia, se participó en la vida diaria de tres familias (dos dedicadas a actividades agrarias y una pecuaria), para recopilar información etnográfica (Russell Bernard, 1995).

Durantecuatrodíasporfamilia, hubounacompañamiento en sus actividades productivas - primordialmente -, donde se priorizaron aquellas actividades que tenían que ver con el riego de sus terrenos en producción. A la par, y después de estos 12 días de convivencia, se hicieron entrevistas a autoridades locales de varias instituciones (comunidad campesina, municipalidad distrital, gobernación). Se usó la técnica de la observación participante, registrando lo vivido mediante anotaciones en una libreta que el investigador llevaba consigo.

También se realizaron observaciones no intrusivas, anotando lo observado en cuadernos de campo y registrando, al final del día, un diario donde se detallaban recuerdos de las interacciones que acontecían durante el día (además de ideas o pensamientos considerados claves por el investigador). Durante estas observaciones, también surgía la posibilidad de conversar con personas que se encontraba en ese lugar, a esa hora, dando pie a varios episodios entretenidos e inesperados de interlocución.

Se realizaron un total de 10 entrevistas en profundidad; una parte de ellas se efectuaron con miembros de las familias con las que se convivió. Otras se hacían con personas referidas por estas familias, ya sea porque eran autoridades cuya pertinencia en el tema hacía necesario su intervención en el estudio o porque el propio investigador consideró necesario entrevistarles. Se grabaron las entrevistas en una grabadora digital, no sin antes solicitar el permiso de los entrevistados para hacerlo. En aquellos casos donde no se dio este permiso, no se grabó el diálogo 
(como sucedió con el caso del tesorero de la comunidad campesina).

El análisis se realizó en varias etapas. La primera tuvo que ver con la organización de toda la información recopilada. Por ejemplo, una vez transcritas todas las entrevistas, se elaboró un cuadro Excel donde se colocó la data extraída de los testimonios que se relacionaban con los temas que se colocaban en cada fila. De esta forma, en cada fila había una temática distinta, mientras en cada columna se colocaba la información de un mismo entrevistado. Al final, este cuadro permitía dos cosas: A. Analizar la información de un solo entrevistado; y B. Cruzar la información de ese entrevistado con el de otros entrevistados, información que también se había colocado en ese mismo cuadro.

Se registraron varias fotografías que sirvieron como material visual, que apoyaron en la reconstrucción de la descripción de ciertos eventos y elementos del distrito. También, se utilizó información de fuentes secundarias censos agropecuarios - para sostener ciertos argumentos, contrastar con la información recopilada y permitir un cruce que evite mayores sesgos en el análisis. Esta información se puede observar en los cuadros que se presentan en este texto.

\section{Resultados y discusión}

\section{Contexto local}

San Andrés de Tupicocha es un distrito de la provincia de Huarochirí, ubicado en la cuenca alta del río Lurín, a tres horas de la ciudad de Lima. Se encuentra situada entre los 1800 y 4700 msnm. La superficie total del mismo es de aproximadamente $84 \mathrm{~km}^{2}$. Fue declarado como distrito en 1943, aunque pre-existe la Comunidad Campesina de San Andrés de Tupicocha, entidad que actualmente organiza las familias Tupicochanas en barrios o ayllus (Municipalidad Distrital de San Andrés de Tupicocha, 2012 ). Colinda con otros 5 distritos: Por el norte con Surco y San Bartolomé, por el este con Santiago de Tuna, por el sur con Antioquía y por el oeste con San Damián. Entre los censos del 2007 al 2017, el total de la población disminuyó de 1453 a 1320.

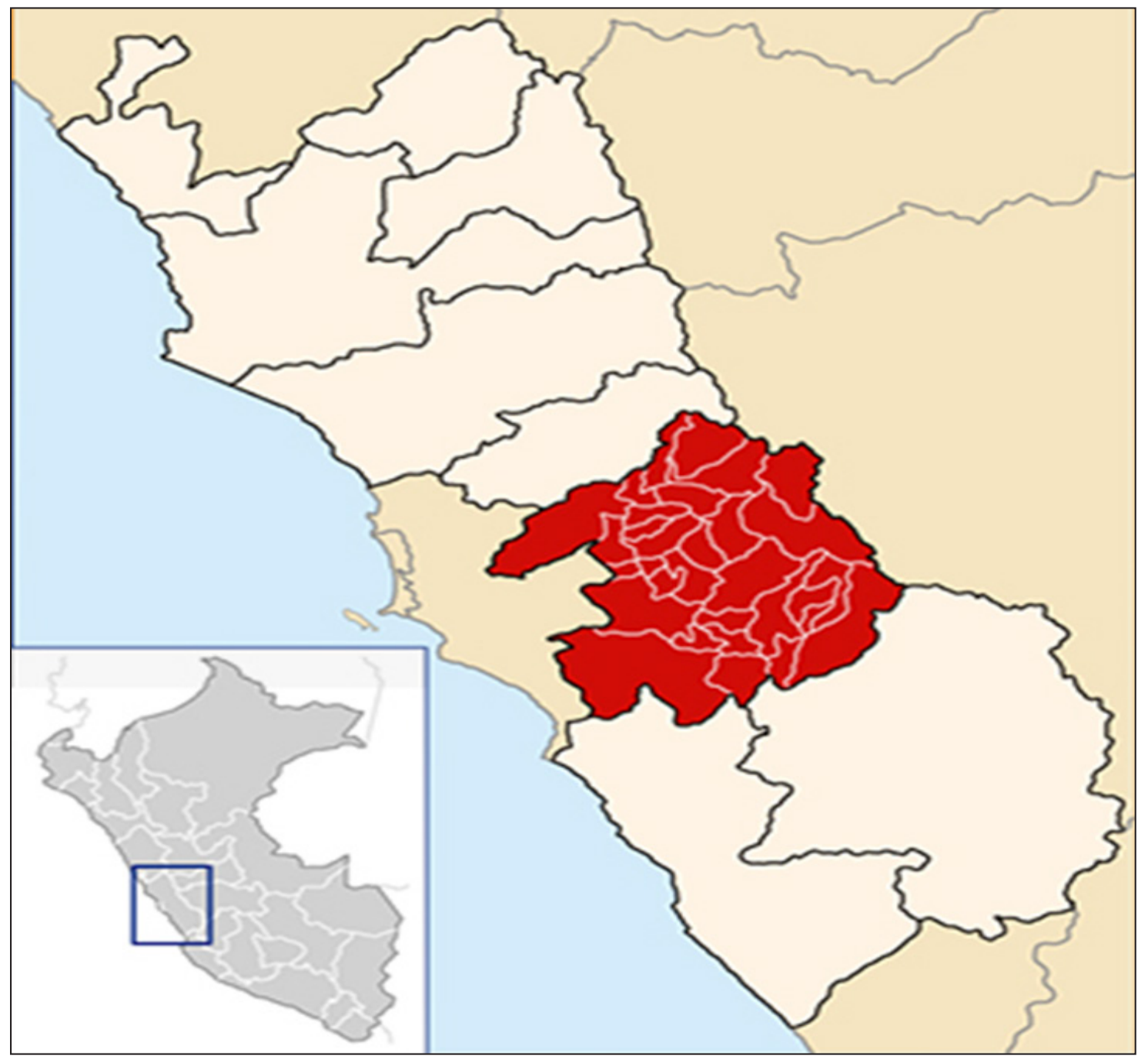

Figura 1. Mapa de la región Lima, provincia de Huarochirí (rojo), distrito de San Andrés de Tupicocha (punto amarillo) 
San Andrés de Tupicocha sigue siendo un distrito “joven": los grupos etarios con mayor número de miembros están entre los 0 y los 18 años de edad - infantes, niños y niñas ( 0 a 9 años), junto con adolescentes (10 a 19 años de edad). En comparación con los grupos etarios que van desde los 20 en adelante, Según algunos de los entrevistados se movilizaron (migraron) hacia Lima, la capital del país, en respuesta a la precariedad de la situación socioeconómica del distrito, optando por buscar mejorar sus vidas, hay una notable disminución en la cantidad de población que vive en Tupicocha actualmente, particularmente las mujeres que suelen tener números más bajos, en comparación a los varones, en los grupos etarios más numerosos (Figura 2). visitas servían para entablar conversaciones con personal técnico y especializado en dicho centro. En varias de estas conversaciones, el personal mencionó que el distrito sufría de altos índices de anemia y DCI, en niñas y niños menores de cinco años.

Tupicocha también es un distrito considerado por la PNUD con niveles de desarrollo muy por debajo de lo establecido en el criterio de Bajo Desarrollo Humano. El IDH del 2012 para este distrito se estimó en 0,2151; colocándolo como el tercer distrito menos desarrollado en la provincia de Huarochirí, con una esperanza de vida

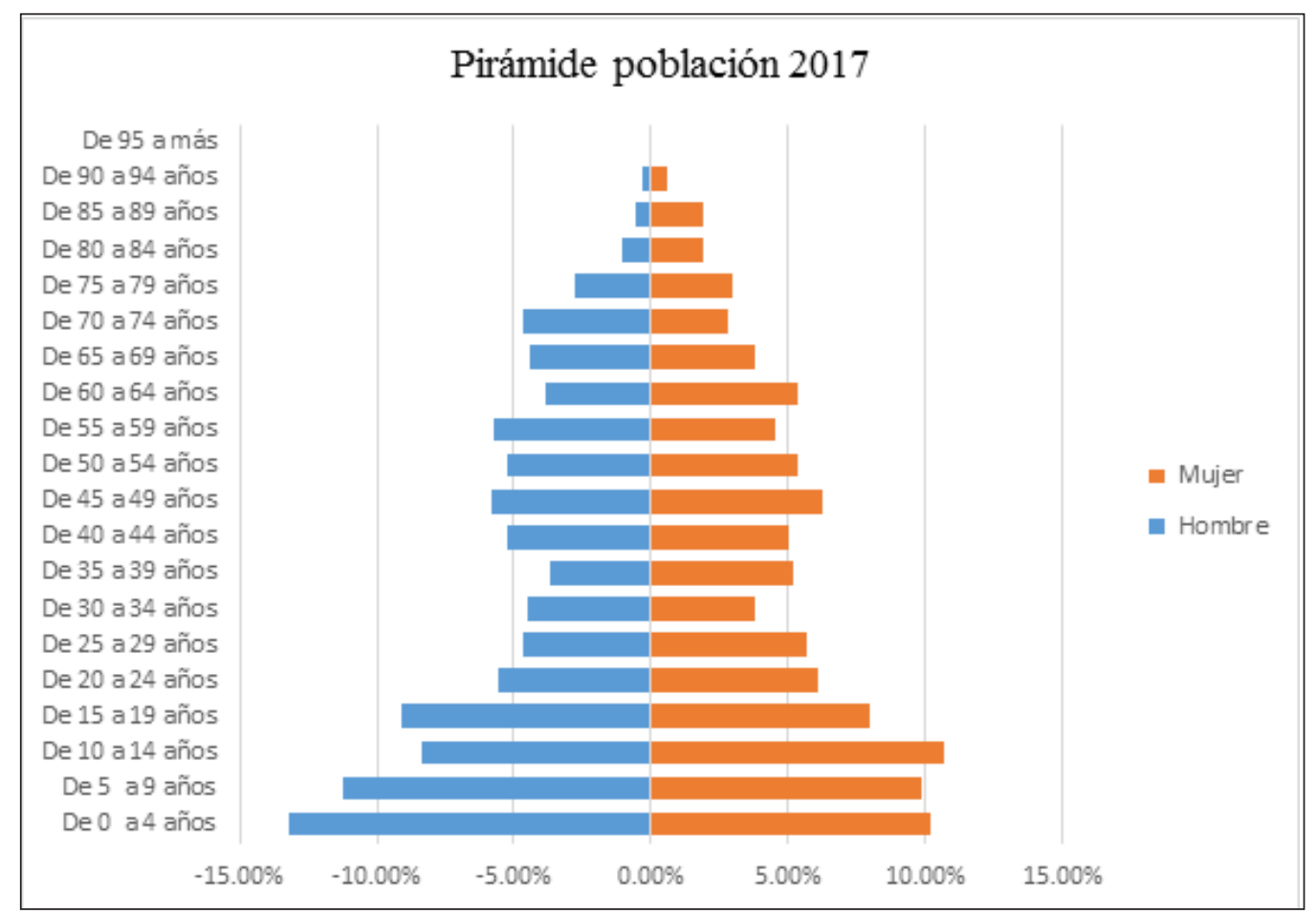

Figura 2. Pirámide poblacional San Andrés de Tupicocha - 2007

El grupo etario más vulnerable a los problemas nutricionales era, a la vez, el grupo etario más numeroso en el distrito de Tupicocha. Según el Mapa de Desnutrición Crónica en Niñas y Niños menores de cinco años a nivel provincial y distrital (INEI, 2009), San Andrés de Tupicocha es el distrito con mayor proporción de niñas y niños con DCI $(75 \%)$. Este dato coloca a Tupicocha como el distrito en todo el departamento de Lima con el porcentaje más elevado de niñas y niños menores de cino años con este mal.

Contrario a esta situación en Tupicocha, el MINSA confirmó que - a nivel departamental - la DCI ha tenido un descenso sostenido desde el 2010 (8,9\%) hasta el 2014 (4,8 \%). Según datos del ENAHO (2013), a nivel nacional, el departamento de Lima se encontraba en el antepenúltimo lugar en incidencia de la DCI. Decía que 5,1\% de toda la población menor a cinco años en el departamento de Lima aún tenía algún indicio de sufrir este mal (ENAHO, 2009). Durante el trabajo de campo se hicieron varias visitas al centro de salud de la localidad de Tupicocha. Estas media a nivel provincial (73,8 años, 15 de 32 distritos de la provincia de Huarochirí).

Sobre educación en Tupicocha, el IDH muestra un bajo porcentaje de la población distrital que culminó su secundaria $(25,20 \%$, cuarto distrito más bajo a nivel provincial). Es el tercer distrito con el peor nivel de años de escolaridad por poblador en la provincia de Huarochirí (promedio de 6,4 años de escolaridad) y el quinto distrito, a nivel provincial, con el ingreso familiar per cápita más bajo (S/.141,00 por mes/poblador).

Para el censo del 2017, casi la totalidad de los pobladores censados residían en el distrito. De hecho, solo $1,2 \%$ (16 pobladores) del total de la población no vivía en Tupicocha, retornando ocasionalmente. Al comparar estos datos con aquellos del año 2007, se observa que hubo una disminución en la población, lo que promueve la idea de movilizaciones migratorios de pobladores que salen de ese distrito, rumbo a otros espacios (Lima es el foco de atracción más grande). 
$\underline{\text { Innovación en sistemas de riego y cambios en producción }}$ agraria

Para muchas familias en Tupicocha, el sistema de represas comunales construidas hace más de 20 años (Durand, 2011) significó el acceso a una mayor cantidad de agua, especialmente para sus actividades productivas. Este sistema otorgaba agua, principalmente, durante las épocas de estiaje (cuando las lluvias ya no caen). Esto significó que las actividades productivas que más se desarrollaban los pobladores en la zona (la agricultura, ganadería y silvicultura) ahora se podrían realizar casi todo el año.

Antes de la construcción de estas represas, la agricultura se trabajaba por secano (acceso al agua mediante lluvia), donde la población producía cultivos considerados por algunos entrevistados como "tradicionales". Al realizar el contraste de este cambio percibido con información de los censos agropecuarios de 1993 y 2012, se corroboró el paso de una agricultura de "secano" a una de "riego": la plantación de ciruela; en esta..., no habia poblado de ciruela, toda esta parte para abajo no había. Imaginate, ha crecido, y acá, en la parte alta, arribita nomás, hay una pampa grande y todo eso ya se ha convertido en ciruela." (DRJ - Anterior autoridad de la Comunidad Campesina de San Andrés de Tupicocha)

Varios entrevistados manifestaron que las familias Tupicochanas estaban acostumbradas a consumir productos que ellos mismos cosechaban: Papa, olluco, oca, trigo, mashua, cebada, zanahorias, entre otros. Según testimonios, la población ahora prefiere consumir productos provenientes de Lima, que se compran en tiendas, cuya presencia en Tupicocha se ha venido aumentando. Los testimonios fueron corroborados con datos de los censos agropecuarios de 1993 y 2012: se sustituyeron los productos antes mencionados por otros como la alfalfa, los frutales, las manzanas, las ciruelas y/o la tuna, tal como se puede observar en la Figura 4.

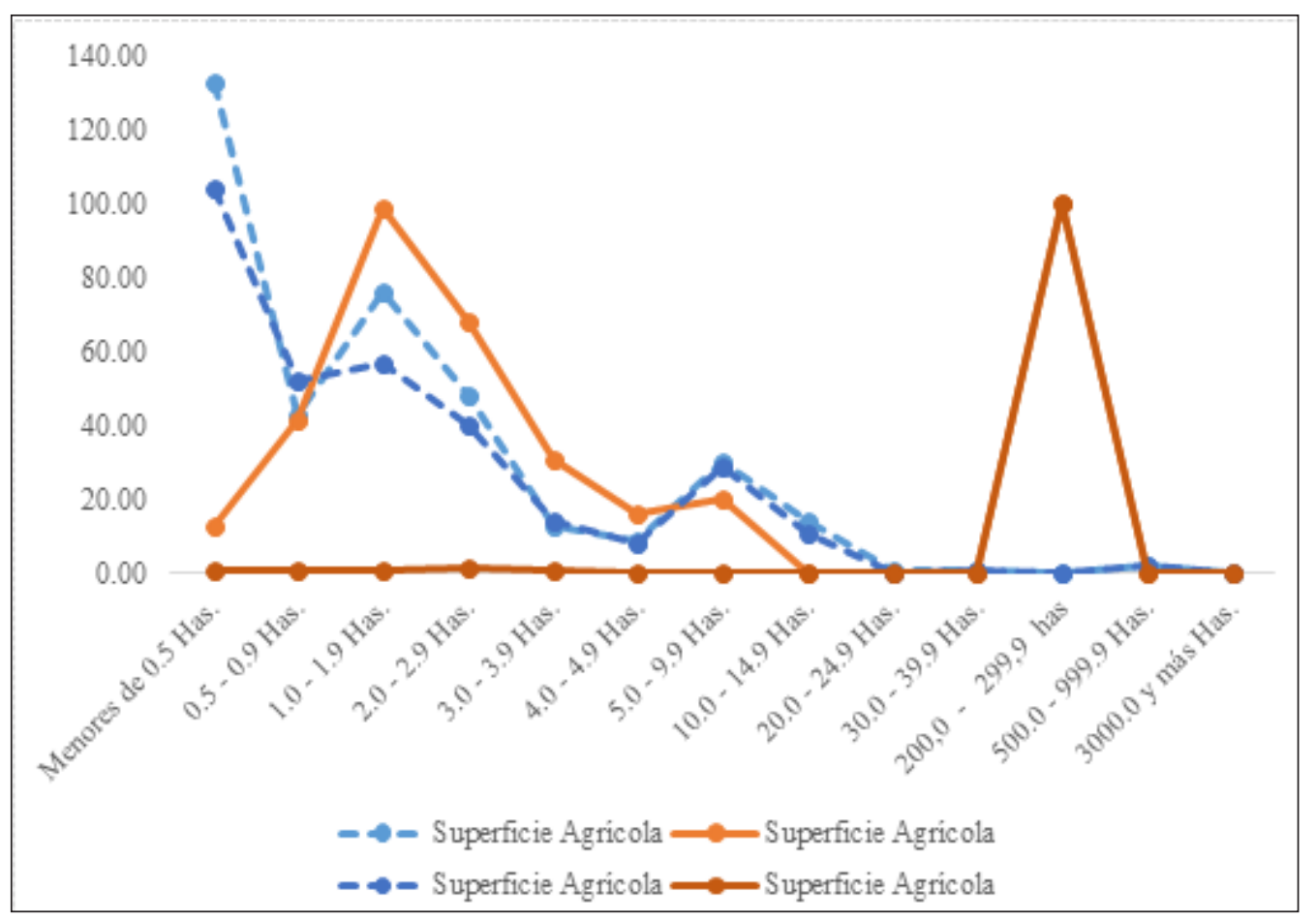

Figura 3. Cambio en acceso y uso de agua para agricultura - 1993 a 2012

Para el año 1993 aún persistían familias con terrenos entre los 0,5 y 25 hectáreas, que recibían agua a través de la lluvia. Para el 2012, las superficies donde se desarrollaba agricultura y que recibían su agua por secano, ya no existían. De este primer cambio surgieron otros. Uno en particular llamó la atención por su importancia para la alimentación familiar: la llegada del sistema de represas comunales modificó indiscutiblemente las prácticas productivas agrarias:

"El cultivo ya cambia pues, como le decía, cuando ya tenemos el aprovechamiento de la laguna o las represas, o del canal que ha llegado de Willcapampa ya se dio otros cultivos ya. Muchos optaron por hacer
Entre ambos censos se nota una clara tendencia de cambio de cultivos: Primero, se dejan de cultivar productos para el autoconsumo, para favorecer o sustituirlos por productos que están destinados a circuitos comerciales, principalmente el mercado limeño (Figuras 5 y 6 ). Segundo, esto significó una reducción en la diversidad de principales productos que se cultivan; se comenzó a instaurar el monocultivo en Tupicocha. En tercer lugar, a la vez se ampliaron - y por tanto se diversificaronnuevas actividades productivas en Tupicocoha, dando pie a la silvicultura (sembrío de árboles) con la plantación de eucalipto, pino y los propios árboles frutales, asociados a las actividades agrarias actuales. 


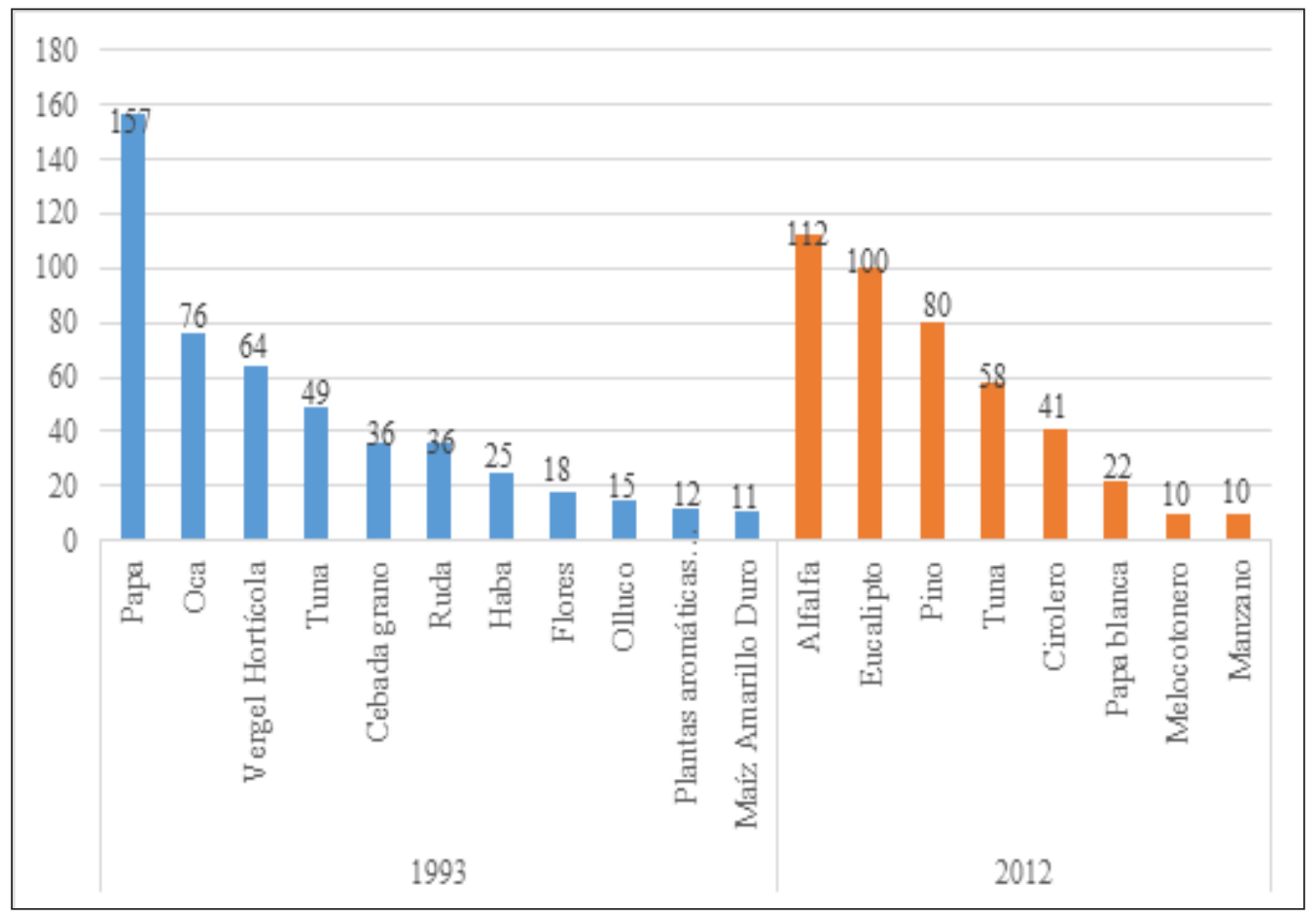

Figura 4. Total de Cultivos Principales entre III y IV Censos Agropecuarios (1993 - 2012)

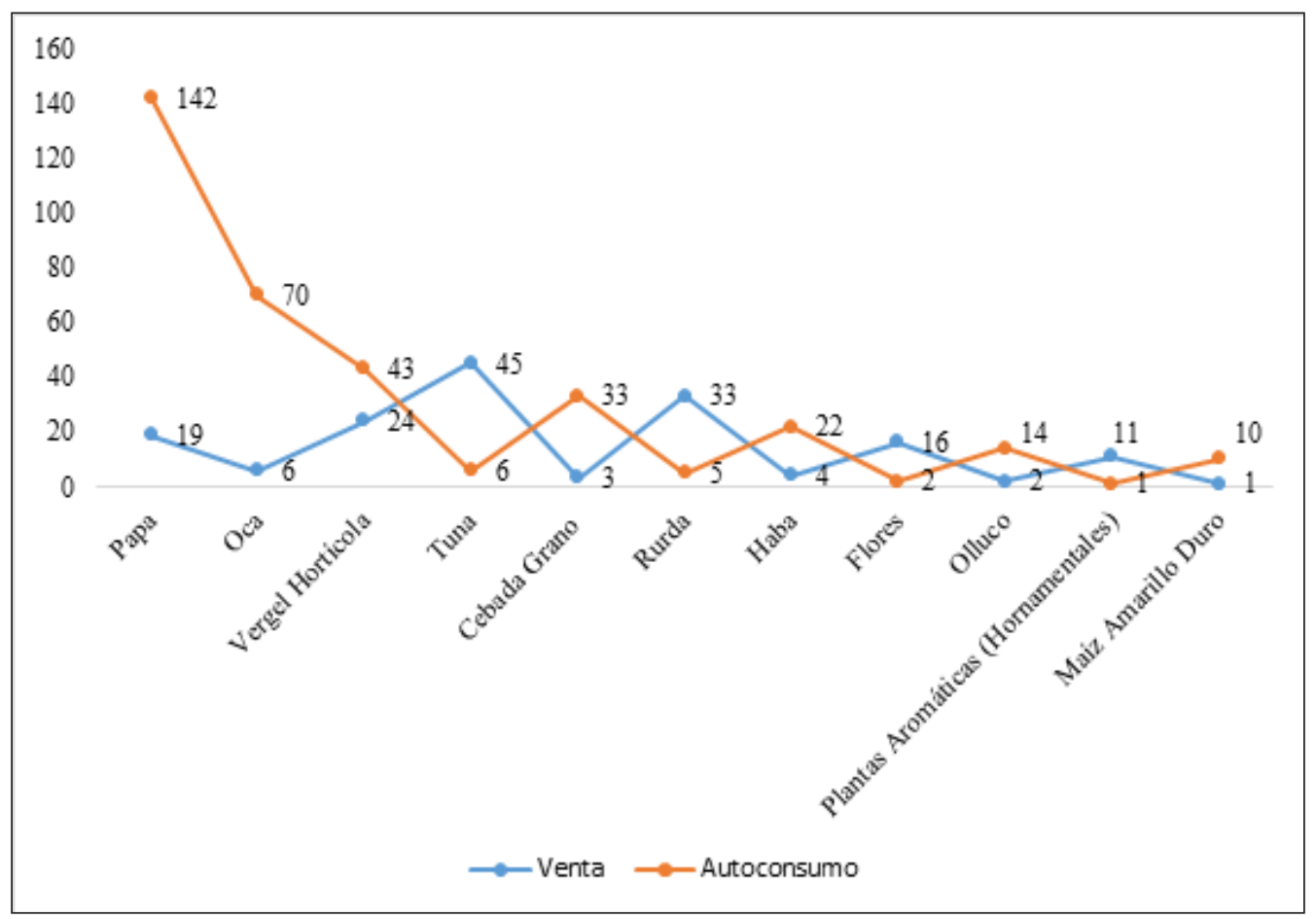

Figura 5. Destino de producción agrícola - 1993 


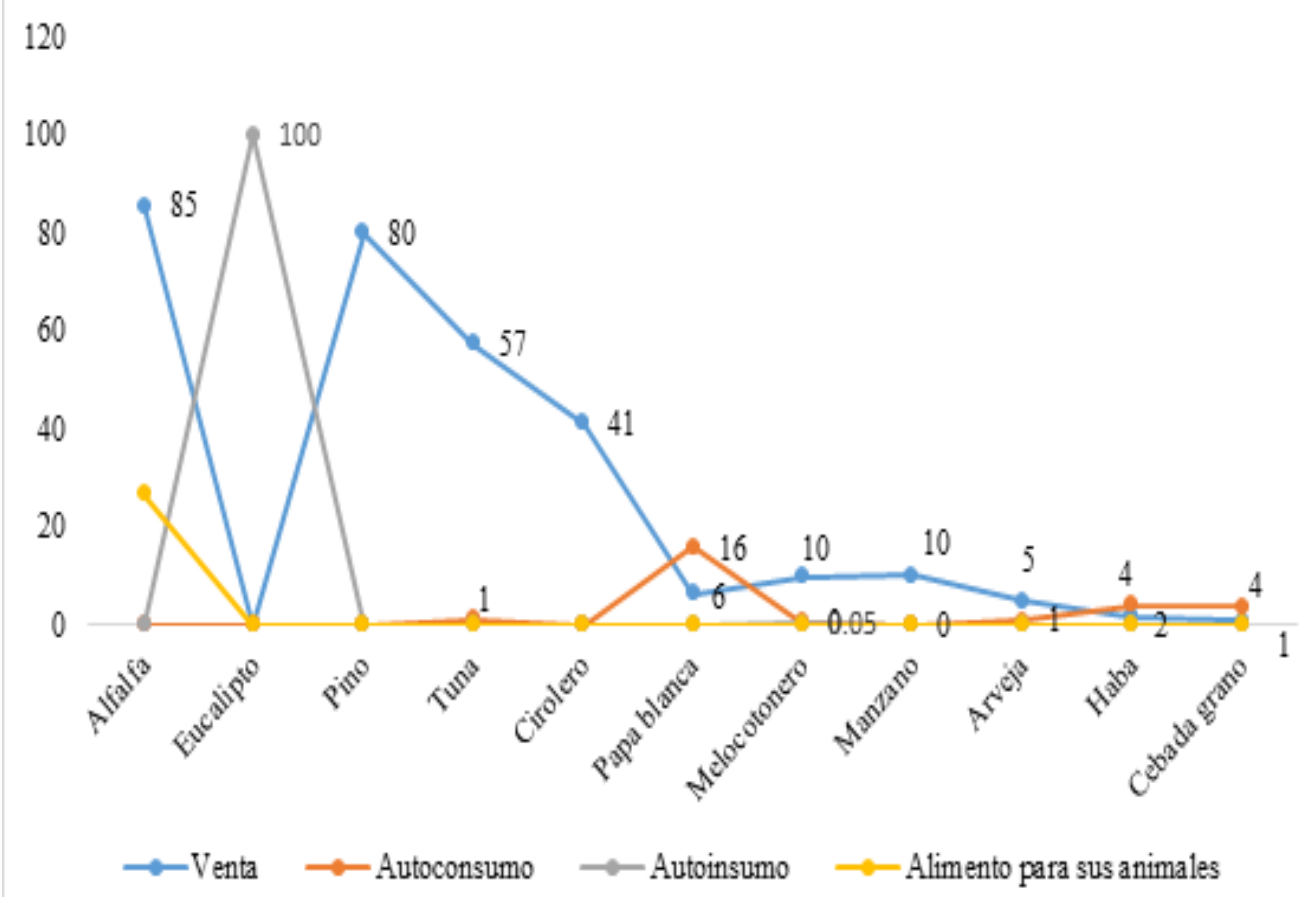

Figura 6. Destino de producción agrícola - 2012

Percepción sobre cambios en la alimentación de familias en Tupicocha

La llegada del nuevo sistema de riego motivó cambios en la producción agraria. Estudios como el de Lía Soto, Iván del Callejo (ambos en Bolivia) y Omar Varillas (Perú) también muestran la cercana relación existente entre agua y alimentación, particularmente desde las perspectivas de las seguridades (hídricas y alimentarias), donde la idea del acceso al agua también afecta el acceso a los productos agrarios que pequeños agricultores en Ecuador, Perú y Bolivia estarían cosechando. (Riego campesino en los Andes: seguridad hídrica y seguridad alimentaria en Ecuador, Perú y Bolivia.(Vos y Román, 2010). Al comenzar el texto se planteó la posibilidad que esta relación cambiante podría haber ejercido algún tipo de efecto sobre la situación de la alimentación de las familias en Tupicocha. Este viraje de la producción agrícola para el autoconsumo hacia una producción dedicada a sostener los mercados en Lima no pasó desapercibido por la propia población de Tupicocha:

“(...) porque nosotros estamos viendo ahi en el día, por ejemplo, que Tupicocha, el poblador tupicuchero tiene más ingreso económico, pero imagínate que los alumnos están más desnutridos y más desnutrición crónica... creo que un $70 \%$ de desnutrición crónica, eso está comprobado. " (CRA - Gobernador del distrito de Tupicocha).

El aumento de ingresos monetarios es también producto del cambio en los cultivos agrarios de Tupicocha: se reemplazaron aquellos cultivos que anteriormente alimentaban a familias locales por otros que se compran en tiendas y se perciben como menos nutritivos.
De acuerdo a los señores CRA y JMA, los hábitos alimenticios de las familias en Tupicocha también cambiaron, deteriorándose y causando serios problemas en la salud de la población más joven del distrito. Este deterioro provendría del consumo de alimentos que no son "naturales" (y por ende con poco valor nutricional), que ahora llegan con mayor frecuencia al distrito y que incluso son solicitados por un gran número de familias:

“(...) Antes la alimentación era más natural, se comía bastante papa, se comía bastante oca, se comía bastante olluco, cosa que hoy día ya no, ya no hay esos alimentos, ya no ya. Hoy día mayormente, mayormente el alimento tupicuchano es el pollo, el pollo y su arroz, sus fideos, su quaker, por ejemplo mi abuelita, mi abuelita falleció de 97 años. Ella me contaba que en toda su vida ella nunca comió el arroz, ella en vez de arroz preparaba el trigo pelado, o sea, hacian hervir el agua, lo echaban el trigo al agua hervida y después de un lapso de tiempo lo sobaban" (CRA - Gobernador del distrito de Tupicocha).

“A lo que Lima (...) pollo, mismo pollo pura hormona, pero la gente qué hace..., la gente es más débil, porque de acá..., a la edad mío, por ejemplo, ya vamos a llegar a los 60, 70 años, en cambio mis padres, 80, 90, 96, tengo mi suegra que tiene 96 años y tranquilo, está en Lima, pero está tranquila... De acá, pero como digo, lo de antes era lo de acá, sacaban bastante y natural, todas las cosas de acá. Según ella me cuenta que solamente le compraban su azuquitar y nada más, su arrocito a veces, nada más, todo era todo de acá, no había pan ..., no ha habido." (JMA - Poblador mayor de Tupicocha). 
La alimentación "natural" y que era "anterior" a los sistemas de represas, se estaría dejando de lado por una mejora económica - un incremento en el acceso al dineroproducto del cambio de cultivos y su venta a los mercados en Lima. Lo que se hace explícito es el cambio en ocurrido en la dieta de estas familias: anteriormente cocinaban productos provenientes de sus campañas agrícolas; hoy en día, los productos provienen de las tiendas cercanas a las viviendas ubicadas en el distrito.

Del Pino et al. (2012) dan cuenta de un proceso de cambio en las cuatro comunidades campesinas visitadas en Ayacucho durante su estudio. Proponen que hubo una transformación de modelo o paradigma de crianza, producto del terrorismo y la inclusión de relaciones mercantiles con la expansión del neoliberalismo en Ayacucho: del "modelo cultural de crianza anclado en la fortaleza física" -que requiere de una alimentación acorde, con productos considerados aptos para ello-, hacia una centrada en "las habilidades e inteligencia de los niños" — que se fomenta a través de la enseñanza escolar (Este último factor mueve a muchos padres de familia llevar sus hijos hacia la capital del país o centros urbanos cercanos a las comunidades rurales. Una situación parecida se puede observar en Tupicocha, donde la movilización de los comuneros es motivado por las mejoras en su calidad de vida; la ciudad de Lima es el centro de atracción de esta población.)-

Similar a la situación observada en Ayacucho, padres de familia de Tupicocha (mediante su creciente capacidad financiera) promovieron la búsqueda de una mejor vida para sus hijos, lejos de su comunidad y de las labores agrarias, prácticas tradicionalmente realizadas por la población de ese distrito. Junto a este cambio en la crianza y la idea de desarrollo-bienestar que se estaría implícito, también se dio un cambio en la dieta de la población local.

Elementos subyacentes en la percepción y corroboración de estos cambios

A la vez y casi de manera desapercibida, hay un proceso de transformación que alude al paso de lo antiguo -lo anterior, lo tradicional-, hacia lo nuevo - lo moderno y el prestigio adherido a estos cambios (Kuper et al.; 2017) - Se observa en el siguiente testimonio el relato de abandono de un sistema de riego tradicional (amunas) y los terrenos donde históricamente se labraban estos cultivos en Tupicocha:

"Siempre ha dado beneficio y siempre hay que ser amuna, porque si no los subsuelos se van a secar, nos decian, hay que mantener las amunas y esas cosas tienen que hacer toda la comunidad... Un poco que no ya, poco a poco más gente ya tiene..., unos se van a Lima y todas esas cosas (se vienen dejado de lado)" (BA - Presidente APAFA).

En las premisas presentadas en el libro Dando la Talla del Banco Internacional de Reconstrucción y Fomento (BIRF/BM, 2017), la DCI en el Perú se ha reducido dramáticamente en un periodo un poco menor a diez años. Cuando se pone frente a frente con las percepciones de malnutrición de pobladores de Tupicocha, aparece un contraste. Los entrevistados sostienen, por el contrario, que ahora las niñas y niños de su comunidad están sufriendo más producto de los cambios en la producción agraria acaecidos con la llegada del nuevo sistema de represas comunales. Es más, los datos sobre malnutrición (DCI, anemia y obesidad) que se observan en el acápite de contexto, fundamentan la preocupación de los entrevistados al hacer notar que, en efecto, siguen existiendo niveles elevados de malnutrición en el país, más aún, en Tupicocha.

¿Qué significa este contraste entre los elevados niveles de malnutrición en Tupicocha y las premisas y datos proveídos en el libro Dando la talla (Marini y Rokx; 2017), demostrando una disminución (y tendencia a la disminución) de la Desnutrición Crónica Infantil? Varias ideas surgen aquí.

En primer lugar, hay un escalamiento o "spiraling-up" (Emery y Flora 2005) entre las dos variables de sistema de riego y labores agrícolas, donde la primera afecta de forma positiva a la segunda, haciendo suponer que se percibe una mejora por parte de los entrevistados y la población local. Esta mejora o escalamiento también se ha reproducido en otros aspectos de la vida local tupicochana (Gómez de la Torre, 2017). Sin embargo, para el caso nutricional y la dieta local, más bien parece haberse dado el efecto contrario: de acuerdo a la información cuantitativa encontrada, disminuyó en algo la DCI pero no lo suficiente; en cambio sí hubo un aumento del sobrepeso y obesidad de niñas y niños menores de cinco años.

No se parece haber tomado en consideración efectos distintos a las mejoras más inmediatas. Por ejemplo, la llegada de este nuevo sistema fue pensado como una respuesta a las continuas sequías y la falta de agua para riego, incrementando la productividad. La alimentación familiar, por otro lado, no parece haberse considerado dentro de los supuestos efectos que ocurrirían a las familias en Tupicocha, arriesgando la salud de los más pequeños y la dieta familiar.

En segundo lugar, la relación planteada entre las variables permite pensar a la malnutrición como un problema que va más allá de las prácticas nutricionales familiares únicamente. Como Del Pino et al (2012) establecieron en su libro, se hace imprescindible entender el contexto que envuelven estas prácticas alimentarias para dar cuenta sobre por qué hay niñas y niños que sufren de estos males.

En este caso, entran en escena los procesos de innovación que trajeron consigo la construcción del sistema de represas comunales. Dicho sistema influenció la alimentación familiar al cambiar la forma en que los productores cultivaban: qué cultivaban, cómo cultivaban, qué hacían con esos cultivos y por qué cultivaban.

La última pregunta es la más interesante en tanto que se ha demostrado el motivo por el cual un gran número de familias en Tupicocha ahora cultivan el alfalfa, los frutales, incluso los árboles recientemente sembrados (en 
los últimos 10 años): ahora se trabaja la tierra para vender la producción.

A su vez, parece existir (porque no hay data suficiente para concluir) que, al igual que lo ocurrido en el trabajo de Del Pino et al. (2012), también hay un cambio de modelo de crianza que afectó la situación de alimentación infantil de Tupicocha: ya no se estaría primando la fortaleza del comunero, alistándole para el trabajo agrario en el campo, trabajo que requiere de mucha fuerza. Por el contrario, el desarrollo de las niñas y niños en Tupicocha pasa más por un proceso de "profesionalización", conseguido mediante una educación que se encuentra direccionada a la migración hacia las ciudades más cercanas, en este caso Lima.

Finalmente, habría que revisar el documento elaborado por Marini y Rokx (2017) para rescatar las secuencias positivas que servirían para futuras ocasiones. Sin embargo, este libro hace un recuento general de la DCI, sesgando con un halo positivo la lucha que el país ha venido dando frente a este mal. No cuestiona el trabajo que se ha venido desarrollando con los padres de familia, no considera que (re)afirmar la idea que ellos — los padres - no sabían lo que estaban haciendo, muestra un desentendimiento de las diversas realidades culturales que existen sobre la malnutrición, en el país.

\section{Conclusiones}

El texto haplanteado la existencia de relaciones corroboradas entre diferentes factores, cuya correspondencia ha significado una serie de cambios percibidos por pobladores y que se encuentran en casi toda la población distrital. De esta forma, la inclusión de un nuevo sistema de riego afectó la producción agrícola: sobretodo en torno a los productos que ahora se cultivan, el destino que estos tienen (previamente eran para autoconsumo; ahora son para la venta) y los motivos por los cuales se cultiva. Producto de estos cambios corroborados, se percibió una afectación en la alimentación y en la dieta de familias tupicochanas. Se ha corroborada una mejora en los ingresos monetarios de muchas familias en Tupicocha. Esta mejora, producto de los cambios en las prácticas agrarias, supone también que: 1) ya no se cultiva lo que se consume (modalidad de acceso a los alimentos y calidad del producto); 2) ahora, se tiene la posibilidad de comprar la comida en tiendas (modalidad de acceso).

La relación corroborada y percibida entre sistemas de riego innovadores, afectación en producción agraria, y cambios en la alimentación y dieta, enmarca la situación de la malnutrición dentro de procesos mayores que la mera forma en cómo se alimentan los niños y niñas en este distrito.

Hay varios aspectos que requieren de una mayor profundización, como sería la existencia de relaciones que afectan la alimentación y la dieta familiar en Tupicocha y otros distritos. Sería importante considerar, para futuras evaluaciones de impactos asociados a la construcción de represas, que se comience a incluir variables y factores que afectarían la nutrición infantil y la dieta familiar, tal como se viene describiendo en Tupicocha.

\section{Agradecimientos}

El mayor agradecimiento va para las familias que permitieron su acompañamiento durante el trabajo de campo en Tupicocha; a los transeúntes que se detenían para responder preguntas; a las autoridades que abrieron las puertas a las instituciones locales, ayudando a que este proceso de investigación se pudiera realizar.

\section{Literatura citada}

Alencastre, A.; Gómez de la Torre, J. y Marapí, R. 2016. Libro Azul Perú. Una iniciativa multisectorial y ciudadana por el agua en el Perú. Primera edición. Asociación Civil para la Gestión del Agua en Cuencas AGUA-C. 307 - 336 p.

Bernard, H. R. 1995. Research Methods in Anthropology: qualitative and quantitative approaches - 2nd Edition. AltaMira Press. Walnut Creek, California, USA. 585 p.

Carrasco, M.; Gómez de la Torre, J.; Juárez, H. y Warnaars, M. 2008. Agua y salud en la comunidad de Ñaña, Lima - Perú: un enfoque eco-sistémico. Centro Internacional de la Papa, Programa Urban Harvest; Instituto de Investigación Nutricional. $35 \mathrm{p}$.

Creed-Kanashiro, H.; Astete Robillard, L.; Abad Arrue, M.; Marin, M. y Bartolini, R. 2014. Línea de Base Nutricional Perú. Centro Internacional de la Papa. Lima, Perú. 65 p.

Durand, P. 2011. Sembrando y cosechando agua. Proceso de adopción tecnológica y gestión del agua en la comunidad campesina de Cullpe: Una experiencia de autogestión campesina y cambio social. Revista Andina, 51(1): 9-41.

Emery, M. and Flora. C. 2006. Spiraling Up: Mapping Community Transformation with Community Capitals Framework. En: Community Development: Journal of the Community Development Society, 37(1):15-20.

Gómez de la Torre Barúa, J. 2018. Innovación, Agua y Medios de vida: Discursos de cambio en pobladores de San Andrés de Tupicocha. Tesis de maestría. UNALM. Lima. 290 p.

Instituto Nacional de Estadística e Informática. Disponibles en: https://www.inei.gob.pe/

IV Encuentro Internacional de Saberes y Haceres de los Pueblos Rurales Andinos. 2012. Manejo Integral del Agua en la Cuenca e Lurín. Municipalidad Distrital de San Andrés de Tupicocha. Lima. Disponible en: http:// www.youblisher.com/p/263268-Manejo-integral-deagua/

Jurado, A. R. 2013. Evaluación Final del Programa de Desarrollo Territorial y Generación de Empleo en la Cuenca del Río Lurín, Lima. Banco Interamericano de Desarrollo. Lima/Buenos Aires. 169 p. 
Marini, A.; Rokx, C. (colaboración P. Gallagher). 2017. Dando la talla. El éxito del Perú en la lucha contra la desnutrición crónica. Banco Internacional de Reconstrucción y Fomento/Banco Mundial. Washington, DC. 58 p.

Ministerio de Salud. 2017. Plan Nacional para la Reducción de la Anemia 2017-2021.

Municipalidad Distrital de San Andrés de Tupicocha. Lima, 2012. Manejo Integral del Agua en la Cuenca e Lurín. Disponible en: http://www.youblisher.com/p/263268-Manejointegral-de-agua/

OMS [Organización Mundial de Salud]. 2017. Malnutrición. Nota descriptiva. Disponible en: http:// www.who.int/mediacentre/factsheets/malnutrition/es/

OMS [Organización Mundial de Salud]. 2018. http:// www.who.int/mediacentre/factsheets/ malnutrition/es/

Del Pino, P.; Mena, M.; Torrejón, S.; Del Pino, E.; Aronés, M.; y Portugal, T. 2012. Repensando la Desnutrición: Infancia, alimentación y cultura en Ayacucho, Perú. Lima, Acción contra el Hambre/ACH-España; IEP. 233 p.

Sistema de Información del Estado Nutricional. Disponible en: http://www.portal.ins.gob.pe/es/cenan/cenan-c2/ vigilancia-alimentaria-y-nutricional/sistema-deinformacion-del-estado-nutricional

Venot, J-Ph; Kuper, M. y Zwarteveen, M. 2017. Drip irrigation for agriculture: Untold stories of efficiency, innovation and development. Disponible en: https://g. $\underline{\mathrm{co} / \mathrm{kgs} / 3 \mathrm{Sg} 7 \mathrm{aT}}$

Vos, J. y Román, P. (editores). 2010. Riego campesino en los Andes: seguridad hídrica y seguridad alimentaria en Ecuador, Perú y Bolivia. Lima/Wageningen. 336 p. 